\title{
Impacts of Breeder Age, Storage Time and Setter Ventilation Program on Incubation and Post-Hatch Performance of Broilers
}

\section{-Author(s)}

\section{Okur $\mathrm{N}^{\prime}$ \\ Eleroğlu H"}

Türkoğlu $\mathrm{M}^{\text {"II }}$

Department of Poultry Farming, Faculty of Agriculture and Natural Sciences, Abant Izzet Baysal University, Bolu/Turkey, E-mail: nezihokur@ibu.edu.tr

"Sarkisla Asik Veysel Vocational High School, Cumhuriyet University, Sivas/Turkey, E-mail: eleroglu@cumhuriyet.edu.tr

III Department of Animal Science, Faculty of Agriculture, Ankara University, Ankara/ Turkey, E-mail: mturk@agri.ankara.edu.tr

\section{Mail Address}

Corresponding author e-mail address Nezih Okur

Department of Poultry Farming, Faculty of Agriculture and Natural Sciences, Abant Izzet Baysal University, Bolu/Turkey

Tel: $\quad$ +905332825739

Email: nezihokur@ibu.edu.tr

\section{-Keywords}

Breeder age, broiler, egg storage, incubation, setter ventilation program.

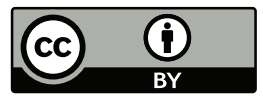

Submitted: 18/May/2017

Approved: 12/September/2017

\section{ABSTRACT}

The impacts of breeder age (32 and 55 weeks), egg storage time (2-12 days), setter ventilation program (control-test) on incubation and post-hatch performance of broilers were investigated in this study. Young $(\mathrm{Y})$ and old $(\mathrm{O})$ breeders' hatching eggs were incubated in two different setters operated by two different ventilation programs as control (C) and test (T). Incubation took place after a short (S) and long $(\mathrm{L})$ time of storage in this study. According to the trial design, eight treatment groups were as YSC, YST, YLC, YLT, OSC, OST, OLC and OLT. In total 9600 eggs and 6400 chicks were used. Early stage embryonic mortality (ED) rates were lower and accordingly hatchability of fertile eggs (HF) were higher $(p<0.05)$ in YS than the other treatment groups. In contrast, incubation performance in eggs hatched in $C$ and $T$ programs were found to be similar. However, interaction between treatments were significant $(p<0.05)$ and ED and HF were significantly $(p<0.05)$ improved at OC. Liveability in growing period was affected $(p<0.05)$ by breeder age but it wasn't affected by storage time and incubation program. Higher post-hatch performance was achieved in chicks of OST but differences between groups weren't significant except for chick weights in comparison by breeder age. However, interactions between treatments were significant $(p<0.05)$ and reached the highest post-hatch performance in YST $(p<0.05)$. In conclusion, the long time storage of old breeders' eggs improved incubation and posthatch performance when they were incubated at test program.

\section{INTRODUCTION}

The hatchery is one of the most important and fragile integral part of the poultry production chain that requires serious amount of investment. The relationship between incubation and growing period began to increase gradually through the use of high-yielding breeds and hybrids. To see the best performance characteristics of broiler chicks in the field, a lot of attention should be paid to the hatchery. In the hatcheries, chick production is carried out based on information about the physiology of embryonic development. The whole immune system and body development of chicks takes place in the hatcheries with contribution of nutritional and genetic aspects of the breeders. Thus the hatchery has an important impact on liveability and post-hatch performance of birds. According to incubation research data (embryonic mortality rate, hatchability of fertile eggs, hatchability etc.) incubation affects the results of the whole production chain. Thus it is very important to analyse and improve the incubation results (Boerjan, 2011).

Incubation performance is affected by genetics, breeder age, egg quality, air pressure, oxygen demand, temperature, humidity, turning, storage time, storage conditions, management conditions of breeders and breeders' health conditions. Performance of the incubation 
sequence can also be determined by economic criteria such as fertility and hatchability of fertile eggs. The post-hatch performance is influenced by factors such as genetics, breeder age, incubation process, management conditions of breeders and condition of the growing house, season and disease. All these factors of management may have some effect on criteria such as average slaughter weight, feed conversion rate, liveability and European productivity index (Turkoglu et al., 2014).

Reaching to the highest incubation performance and achieving high quality chicks in the hatchery depends on reducing deviations in egg quality and the embryonic development. Researches are being taught to understand and identify natural variations in egg quality with embryonic development and some equipment are being developed to provide a uniform incubation. English Buckeye and Belgian Petersime companies suggest the idea that embryonic development may be encouraged via carbon dioxide $\left(\mathrm{CO}_{2}\right)$ accumulation. They developed a technology allowing $\mathrm{CO}_{2}$ to rise for a certain period, then this high level is reduced in a controlled manner by help of infrared $\mathrm{CO}_{2}$ sensor (Elibol \& Turkoglu, 2014).

Researches in the field determined that breeder age, storage time and $\mathrm{CO}_{2}$ levels affected incubation and post-hatch performance. The increase in breeder age (Mather \& Laughlin, 1979; Tona et al., 2004) and storage time (Becker et al., 1968; Butcher 2004; Mather \& Laughlin, 1976; Reis et al., 1997; Tona et al., 2004) negatively affects embryonic mortality (Mather \& Laughlin, 1979; Bruzal et al., 2000; Ross Tech 2003; Elibol \& Brake, 2003) and hatchability of fertile eggs (Mather \& Laughlin, 1976 and 1979; Mc Daniel, 2000; Tona et al., 2004). Generally, embryonic mortality during the incubation period varies between $3-7 \%$ in the early and late stage but it is less than 1\% in mid-term (Romanoff, 1949; Salamon \& Kent, 2014). Embryonic deaths is associated with storage conditions for early stage, malnutrition or infections for mid-term deficits, deficits in incubator and previous periods for last stage (Hodgetts 1993; Bruzal et al. 2000). Depending on embryonic mortality and hatchability of fertile eggs, it is well known that live weight values are also negatively affected by similar conditions (Reis et al., 1997; Tona et al., 2004). Body weight (Christensen et al., 1996), feed conversion ratio (Hill, 2002) and liveability worsens with increasing storage time and breeder age. However, there are studies showing a difference in live weight values especially of first week (Quintana et al., 2000; Tona et al., 2004) this difference closes subsequently (Bowling \& Howarth, 1981; Elibol,
1997). In contrast, different results were obtained through studies on the effects of the $\mathrm{CO}_{2}$ level in the machine (Everaert et al., 2007; 2008).

There are studies that show positive (Ross Tech, 2003) and negative (Taylor, 2000) effects of raising $\mathrm{CO}_{2}$ levels to a certain level or operating $\mathrm{CO}_{2}$ control system (Onagbesan et al., 2007). The first studies on the effects of $\mathrm{CO}_{2}$ during the incubation period reported negative impact on hatchability of chicken eggs when incubator $\mathrm{CO}_{2}$ levels exceeded $1 \%$ during very early incubation (Romanoff \& Romanoff 1933; Barrott, 1937). More recent studies have shown that a gradual increase in $\mathrm{CO}_{2}$ levels up to $1.5 \%$ in the first 10 days of incubation enhanced embryo growth, stimulated early hatching and increased hatchability of chicken and turkey eggs (Gildersleeve \& Boeschen, 1983; Hogg, 1997; De Smit et al., 2006; Tona et al., 2006) and is shown that this effect changed with strain (De Smit et al., 2008; Tona et al., 2013). When ventilation system of the incubator was not operated and the ventilation holes of incubator were closed for this aim, albumen $\mathrm{pH}$ and egg weight loss decreased, embryonic body weight and hatchability increased. Also, earlier hatch (approximately 8,68h) and narrower hatch window was obtained where lesser embryonic mortality (because of a reduction in embryo mal positioning) and higher chick body weight was found. Hormonal levels as triiodothyronine $\left(T_{3}\right)$, thyroxine $\left(\mathrm{T}_{4}\right)$ and corticosterone with records of blood haematology parameters as haemoglobin $(\mathrm{Hb}), \%$ packed cell volume (PCV) and red blood cell (RBC) count were determined therewithal (De Smit et al., 2008; Fares et al., 2011; Tona et al., 2007; Willemsen et al., 2008). At the same time during the post-hatch period, first week (De Smit et al., 2008) final body weight, body weight gain, feed consumption and feed conversion ratio were shown to be slightly higher (Fares et al., 2011). It was concluded that the effects of high $\mathrm{CO}_{2}$ levels were found to be differentiating on incubation and post-hatch performance according to applied levels and times, after the first 10 days of incubation period. It was shown that high (9\%) $\mathrm{CO}_{2}$ after $9^{\text {th }}$ days of incubation increased blood bicarbonate and $\mathrm{pH}$ values (Dawes et al., 1971), high (4\%) $\mathrm{CO}_{2}$ between $12^{\text {th }}-18^{\text {th }}$ days did not change hatching parameters and first week body weight where corticosterone and $T_{4}$ levels were found to increase in contrast (Everaert et al., 2007; 2008).

Studies to provide more uniform hatchability of fertile eggs and lesser distortions in performance are continuing by improvisations of incubation and posthatch conditions. In particular, to determine the effects of applied processes from breeder farms to hatchery 
till the slaughter age, and to produce solutions are becoming more and more important. This research was conducted in the light of these information and it was aimed to reach higher hatchability of fertile eggs and reduce the deviations in performance by determining the effects of breeder age, storage time and ventilation program in the setters.

\section{MATERIALS AND METHODS}

The impacts of breeder age, storage time and setter ventilation program on incubation and post-hatch performance were examined in this study trial design shown on Table 1. For this purpose, percentage of embryonic mortality and hatchability of fertile eggs as a hatchery criteria and liveability, chick weight (CW), body weights on $41^{\text {st }}$ day of growing period (BW), daily weight gain (DWG), feed conversion ratios (FCR), European efficiency index (EPEF) data as field criteria were gathered.

In this study, 9600 hatching eggs obtained from young ( 32 weeks) and old ( 55 weeks) Ross 308 broiler breeders and 6400 broiler chicks (randomly selected to test house compartment capacity from hatched

Table 1 - Trial design and used hatching eggs and broiler chick amounts in the study.

\begin{tabular}{|c|c|c|c|c|c|c|c|c|}
\hline Flock Age & \multicolumn{4}{|c|}{ Young $(Y), 32$ weeks age } & \multicolumn{4}{|c|}{ Old $(0), 55$ weeks age } \\
\hline Egg Number & \multicolumn{4}{|c|}{4800} & \multicolumn{4}{|c|}{4800} \\
\hline Storage Time & \multicolumn{4}{|c|}{ Short (S) } & \multicolumn{4}{|c|}{ Long (L) } \\
\hline Egg Number & \multicolumn{4}{|c|}{4800} & \multicolumn{4}{|c|}{4800} \\
\hline Ventilation Programme & \multicolumn{4}{|c|}{ Control (C) } & \multicolumn{4}{|c|}{ Test (T) } \\
\hline Egg Number & \multicolumn{4}{|c|}{4800} & \multicolumn{4}{|c|}{4800} \\
\hline Treatment Groups & YSC* & YSN* & YLC* & YLN* & OSC* & OSN* & $\mathrm{OLC}^{*}$ & OSN* \\
\hline Egg Number & 1200 & 1200 & 1200 & 1200 & 1200 & 1200 & 1200 & 1200 \\
\hline Chick Number & 800 & 800 & 800 & 800 & 800 & 800 & 800 & 800 \\
\hline
\end{tabular}

* YSC: Young flock, short storage time, control setter ventilation programme. YSN: Young flock, short storage tine, different (test) setter ventilation programme. YLC: Young flock, long storage time, control setter ventilation programme. YLT: Young flock, long storage time, different test setter ventilation programme. OSC: Old flock, short storage tine, control setter ventilation programme. OST: Old flock, short storage time, test setter ventilation programme. OLC: Old flock, long storage time, control setter ventilation programme. OLT: Old flock, long storage time, test setter ventilation programme.

healthy day old chicks) were used in this study. Average hen housed egg productions and egg weights of flocks were $82-49 \%$ and $60.88-61.34 \mathrm{~g}$ respectively during the study. The incubation phase of the research was conducted at the hatchery of beypiliç ${ }^{\circledR}$ in Bolu, Turkey. Eggs were set in programmable, fully automatic Petersime 57600 eggs capacity setter and Petersime 19200 eggs capacity hatcher with $\mathrm{CO}_{2}$ controlling system in the hatchery. Growing phase of the research was carried out in beypiliç's ${ }^{\circledR}$ broiler research house and 32 compartments $(2 \times 6.5 \mathrm{~m})$ in this house equipped with pan feeders, nipple drinkers and special radiant heaters were used.

First, hatching eggs obtained from young and old flocks were divided randomly into two equal parts and stored for 2 and 12 days. Eggs were stored at $18^{\circ} \mathrm{C}$ temperature and approximately $75 \%$ of relative humidity. Later, the same eggs were divided into 2 equal parts and randomly placed in two different setters which were operated at two different ventilation programs (Figure 1 and Table 2). The first setter was operated by normal ventilation program (control) $\overline{\bar{i}}$ where the certain level of $\mathrm{CO}_{2}$ in the machine was allowed by deactivating $\mathrm{CO}_{2}$ control system. The second machine was operated by a different ventilation program (test)

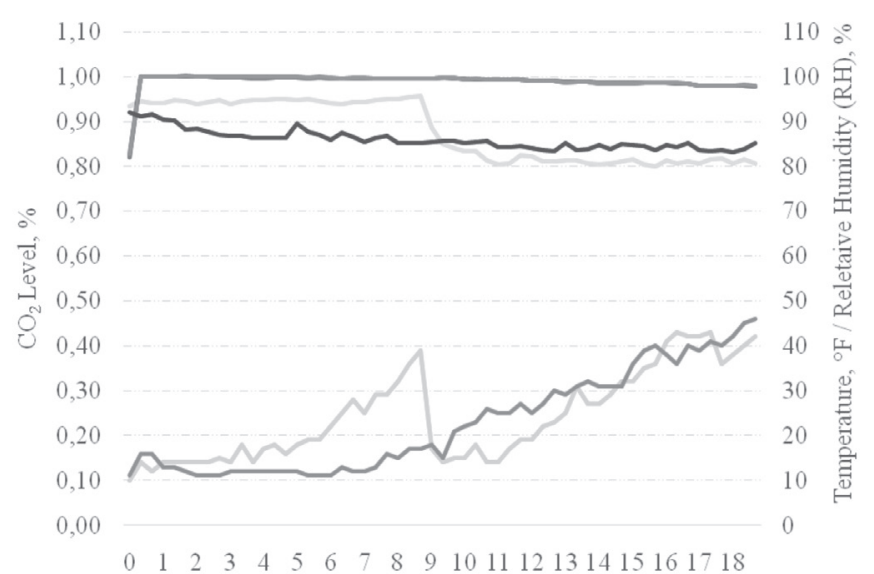

Days of Incubation

- Test — Control — Test — Test — Control—Control

Figure 1 - Actualized temperature $\left({ }^{\circ} \mathrm{F}\right)$, relative humidity $(\%)$ and $\mathrm{CO}_{2}(\%)$ levels during incubation phase in the study.

to provide rapidly accumulating and increasing $\mathrm{CO}_{2}$ levels in the machine up to 9 days and later gradually reducing by using $\mathrm{CO}_{2}$ control system and by arranging the openings of the ventilation flaps. 150 eggs were placed on each setter trays and each tray was accepted as a replicate. Accordingly 32 replications (tray) in total have been used as 8 replications per treatment during the incubation stage of the study. 
Table 2 - Actualized temperature $\left({ }^{\circ} \mathrm{F}\right)$, relative humidity $(\mathrm{RH})$ and $\mathrm{CO}_{2}(\%)$ values in main days of incubation in the study.

\begin{tabular}{lcccccc}
\hline & \multicolumn{2}{c}{ Temperature, ${ }^{\circ}$} & \multicolumn{2}{c}{ Relative Humidity (RH), \% } & \multicolumn{2}{c}{$\mathrm{CO}_{2}$} \\
Incubation days & Control (C) & Test (T) & Control (C) & Test (T) & Control (C) & Test (T) \\
\hline 2 & 99.9 & 100.0 & 87.6 & 96.1 & 0.11 & 0.14 \\
9 & 99.7 & 99.5 & 85.7 & 91.4 & 0.17 & 0.39 \\
18.5 (transfer) & 98.0 & 98.2 & 83.2 & 70.1 & 0.46 & 0.42 \\
\hline
\end{tabular}

Eggs have been placed on different setters till the transfer time (at 18.5 day) and then transferred to the same hatcher and the program recommended by Petersime was applied to all as same. After the completion of the hatching process, culls and unhatched eggs in hatcher trays have been separated for hatch breakout analysis. Early (0-7 day), mid-term (8-17) and late (18-21) stage embryonic mortality rates and pipped but unhatched embryo rates were detected according to Hodgetts (1993) by opening unhatched eggs.

Treatment chicks have been sexed and vaccinated. Chicks arriving the house were weighed and randomly distributed to the pen as 16 chicks $/ \mathrm{m}^{2}$ (200 chicks/ compartment) stocking density and $50 \%$ male $+50 \%$ female for each subgroup. Totally 4 subgroup were used with 4 replications per treatment during the rearing period.

Table 3 - Raw material composition of feed used in the study

\begin{tabular}{|c|c|c|c|c|}
\hline & Chick Starter $^{1}$ & Chick Grower ${ }^{2}$ & Finisher $^{3}$ & Pre-Slaughter ${ }^{3}$ \\
\hline Anticoccidial $^{4}$ & 0.50 & 0.50 & 0.00 & 0.00 \\
\hline Anticoccidial $^{5}$ & 0.00 & 0.00 & 0.50 & 0.00 \\
\hline Barley & 20.00 & 20.00 & 20.00 & 20.00 \\
\hline Broiler Starter Vitamin & 1.00 & 0.00 & 0.00 & 0.00 \\
\hline Broiler Chick Vitamin & 0.00 & 1.00 & 0.00 & 0.00 \\
\hline Broiler Vitamin & 0.00 & 0.00 & 1.00 & 1.00 \\
\hline Choline Chloride-75 & 0.36 & 0.33 & 0.24 & 0.24 \\
\hline DCP-18 & 6.20 & 4.70 & 3.50 & 3.50 \\
\hline Corn & 494.84 & 562.82 & 604.39 & 604.89 \\
\hline Corn Gluten, 60 & 30.00 & 20.00 & 15.00 & 15.00 \\
\hline Full-fat Soybean Meal & 144.56 & 139.59 & 132.67 & 132.67 \\
\hline Lysine-997 & 3.12 & 2.80 & 1.52 & 1.52 \\
\hline Marble Powder & 10.94 & 8.64 & 8.50 & 8.50 \\
\hline $\mathrm{MDCP}$ & 6.50 & 4.50 & 3.80 & 3.80 \\
\hline Methionine, dry ${ }^{8}$ & 2.00 & 1.20 & 1.00 & 1.00 \\
\hline Methionine, liquid ${ }^{9}$ & 1.18 & 1.30 & 1.00 & 1.00 \\
\hline Phytase Enzyme ${ }^{10}$ & 0.30 & 0.30 & 0.30 & 0.30 \\
\hline Poultry Offal Meal & 25.00 & 44.00 & 52.00 & 52.00 \\
\hline Poultry Trace Elements & 1.00 & 1.00 & 1.00 & 1.00 \\
\hline Salt & 2.30 & 2.10 & 2.00 & 2.00 \\
\hline Sodium Bi carbonate & 2.00 & 1.60 & 1.50 & 1.50 \\
\hline Soybeean Meal-48 & 213.90 & 142.32 & 121.68 & 121.68 \\
\hline Vegetable Oil, soybean & 8.00 & 16.00 & 28.00 & 28.00 \\
\hline Wheat & 20.00 & 20.00 & 20.00 & 20.00 \\
\hline Total & $1,000.00$ & $1,000.00$ & $1,000.00$ & $1,000.00$ \\
\hline
\end{tabular}

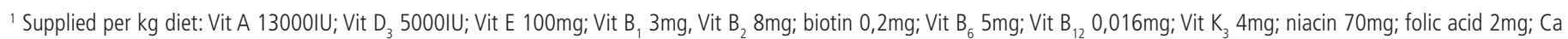
pantothenate 20mg; Mn 120mg; Zn 100mg; Se 0.3mg; Cu 16mg; Fe 50mg; I $2 \mathrm{mg}$ and antioxidant $125 \mathrm{mg}$.

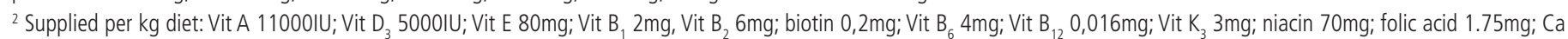
pantothenate 20mg; Mn 120mg; Zn 100mg; Se 0.3mg; Cu 16mg; Fe 50mg; I 2mg and antioxidant $125 \mathrm{mg}$.

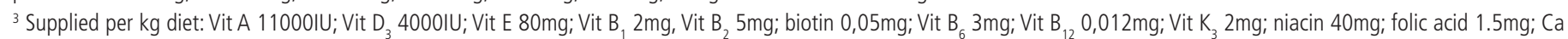
pantothenate 20mg; Mn 120mg; Zn 100mg; Se 0.3mg; Cu 16mg; Fe 50mg; I 2mg and antioxidant 125mg.

${ }^{4}$ Clinacox, anticoccidial (Lly ilac, Istanbul Turkey)

${ }^{5}$ Salinomycin, anticoccidial (Lily ilaç, Istanbul Turkey)

${ }^{6}$ DCP-18 ve MDCP (Rotem Turkey, Istanbul Turkey)

${ }^{7}$ Lysine-99, Lysine HCL 99 (Rotem Turkey, Istanbul, Turkey)

${ }^{8}$ Dry Methionine, DL Methionine Feed Grade; (Evonik Turkey, Istanbul Turkey)

${ }^{9}$ Liquid Methionine, Alimet; (Novus Turkey, Istanbul Turkey)

${ }^{10}$ Phytase enzyme, Phyzyme (Nutriline Feed and Food Additives L.L.C., Istanbul, Turkey) 
ROSS $^{\circledR}(2.0-2.5 \mathrm{~kg}$ ALW) recommendations (Ross Broiler, 2007) and feed were provided by Bolu Feed Mill of Beypi Inc. Chickens were fed in four phase as follows, $0-10^{\text {th }}, 11-22^{\text {nd }}, 23-35^{\text {th }}$ and $36-41^{\text {st }}$ days (Table 3). Feed ingredients and nutrient compositions of compound feeds were analysed to methods were given by AOAC (1990).

Diets were formulated at international standards (NRC, 1994) and grand parent company Feed consumption in each sub-group was determined by weighing the birds at the end of each feeding phase. Liveability was determined by recording the number of dead chicks (mortality) in sub-groups daily. Treatments were designed to the randomized complete block experiment design. The data were evaluated by using one-way ANOVA (factorial design) and analysed using GLM (General Linear Model) procedures of IBM SPSS 22.0 software package program (SPSS, 2013) Statistical differences among the groups were determined by Duncan's Multiple Comparison Test (F-test) (Duncan, 1955).

\section{RESULTS}

Statistical analyses of the data on the incubation and post-hatch performance has been carried out separately and the results are summarized in tables (Table 4, 5 and 6).

Table 4 - The effect of different incubation practices on incubation performance.

\begin{tabular}{|c|c|c|c|c|}
\hline & \multicolumn{3}{|c|}{ Embryonic Mortality } & \multirow{2}{*}{$\begin{array}{c}\text { Hatchability of Fertile } \\
\text { Eggs }\end{array}$} \\
\hline & $\begin{array}{l}\text { Early Stage } \\
0-5 \text { th days }\end{array}$ & $\begin{array}{l}\text { Mid-term Stage } \\
6-17 \text { th day }\end{array}$ & $\begin{array}{l}\text { Late + Pipped } \\
\text { Unhatched }\end{array}$ & \\
\hline & (ED), \% & (MD), \% & $(\mathrm{LPU}), \%$ & (HF), \% \\
\hline \multicolumn{5}{|c|}{ Breeder Age (BA), week } \\
\hline 32 , young $(Y)$ & $5.43 \pm 0.38^{b}$ & $0.86 \pm 0.11$ & $2.62 \pm 0.29$ & $89.90 \pm 0.56^{a}$ \\
\hline 55, old $(0)$ & $8.71 \pm 0.53^{a}$ & $1.07 \pm 0.17$ & $2.74 \pm 0.28$ & $85.40 \pm 0.63^{b}$ \\
\hline \multicolumn{5}{|l|}{ Storage Time (ST), day } \\
\hline 2 , short (S) & $6.26 \pm 0.63^{b}$ & $0.86 \pm 0.13$ & $2.14 \pm 0.22^{b}$ & $89.43 \pm 0.69^{a}$ \\
\hline 12 , long $(\mathrm{L})$ & $7.88 \pm 0.43^{a}$ & $1.07 \pm 0.16$ & $3.21 \pm 0.31^{\mathrm{a}}$ & $85.88 \pm 0.62^{b}$ \\
\hline \multicolumn{5}{|c|}{ Setter Ventilation Program (SVT) } \\
\hline Control (C) & $7.71 \pm 0.67$ & $0.95 \pm 0.16$ & $2.41 \pm 0.17$ & $87.76 \pm 0.71$ \\
\hline Test (T) & $6.43 \pm 0.38$ & $0.98 \pm 0.13$ & $2.95 \pm 0.36$ & $87.55 \pm 0.77$ \\
\hline \multicolumn{5}{|c|}{ Breeder Age X Storage Time } \\
\hline Young 2 days (YS) & $4.34 \pm 0.36^{c}$ & $0.90 \pm 0.15$ & $1.95 \pm 0.30^{b}$ & $91.86 \pm 0.55^{a}$ \\
\hline Young 12 days (YL) & $6.52 \pm 0.52^{b}$ & $0.81 \pm 0.16$ & $3.29 \pm 0.42^{\mathrm{a}}$ & $87.95 \pm 0.64^{b}$ \\
\hline Old 2 days (OS) & $8.19 \pm 0.97^{a b}$ & $0.81 \pm 0.21$ & $2.34 \pm 0.32^{\mathrm{ab}}$ & $87.00 \pm 0.88^{b}$ \\
\hline Old 12 days (OL) & $9.24 \pm 0.45^{a}$ & $1.34 \pm 0.26$ & $3.14 \pm 0.46^{a}$ & $83.81 \pm 0.71^{\mathrm{c}}$ \\
\hline \multicolumn{5}{|c|}{ Breeder Age X Setter Ventilation Program } \\
\hline Young Control (YC) & $5.29 \pm 0.54^{c}$ & $0.90 \pm 0.15$ & $2.43 \pm 0.24$ & $90.52 \pm 0.67^{a}$ \\
\hline Young Test (YT) & $5.57 \pm 0.54^{c}$ & $0.81 \pm 0.16$ & $2.81 \pm 0.52$ & $89.29 \pm 0.89^{a}$ \\
\hline Old Control (OC) & $10.14 \pm 0.82^{\mathrm{a}}$ & $1.00 \pm 0.29$ & $2.38 \pm 0.25$ & $85.00 \pm 0.68^{b}$ \\
\hline Old Test (OT) & $7.29 \pm 0.45^{b}$ & $1.14 \pm 0.19$ & $3.10 \pm 0.50$ & $85.81 \pm 1.09^{b}$ \\
\hline \multicolumn{5}{|c|}{ Storage Time X Setter Ventilation Program } \\
\hline 2 days Control (SC) & $7.38 \pm 1.14^{\mathrm{ab}}$ & $0.67 \pm 0.17$ & $2.52 \pm 0.25^{b}$ & $88.52 \pm 1.15^{a}$ \\
\hline 2 days Test (ST) & $5.14 \pm 0.39^{b}$ & $1.05 \pm 0.18$ & $1.76 \pm 0.33^{b}$ & $90.33 \pm 0.73^{a}$ \\
\hline 12 days Control (LS) & $8.05 \pm 0.74^{\mathrm{a}}$ & $1.24 \pm 0.26$ & $2.29 \pm 0.23^{b}$ & $87.00 \pm 0.82^{\mathrm{ab}}$ \\
\hline 12 days Test (LT) & $7.71 \pm 0.45^{\mathrm{ab}}$ & $0.90 \pm 0.18$ & $4.14 \pm 0.45^{a}$ & $84.76 \pm 0.84^{b}$ \\
\hline \multicolumn{5}{|l|}{$p$ Value } \\
\hline BA & 0.000 & 0.298 & 0.768 & 0.000 \\
\hline ST & 0.038 & 0.297 & 0.006 & 0.000 \\
\hline SVT & 0.103 & 0.908 & 0.174 & 0.838 \\
\hline $\mathrm{BA} \times \mathrm{ST}$ & 0.000 & 0.212 & 0.046 & 0.000 \\
\hline BA X SVP & 0.000 & 0.702 & 0.553 & 0.000 \\
\hline ST X SVP & 0.033 & 0.244 & 0.000 & 0.001 \\
\hline
\end{tabular}

abcd The difference between the averages indicated by different letters in the same column are statistically significant $(p<0,05)$. 
When the data on the incubation performance was analysed, it was found that breeder age affected early embryonic mortality (ED) and hatchability of fertile eggs (HF) $(p<0.05)$. ED values of young flocks was lower than older one and accordingly HF was higher. It was found that increasing storage time affected ED and late stage embryonic deaths plus pipped and unhatched embryo rate significantly $(p<0.05)$. Because of higher ED and late stage embryonic deaths plus pipped and unhatched embryo rate, lower HF was obtained from 12 days stored eggs than the ones stored for 2 days $(p<0.05)$. No significant differences between ventilation programs have been found about the criteria. Also, interactions between breeder age-setter ventilation program, storage timesetter ventilation program were significant $(p<0.05)$ statistically. It was detected that an improvement were provided in early stage embryonic deaths when hatching eggs of old breeders were incubated in setters operated to test the ventilation program of accumulated $\mathrm{CO}_{2}$ (Table 4).

Post hatch performance data obtained from old breeders' hatched chicks has been achieved numerically higher from the accumulated $\mathrm{CO}_{2}$ ventilation program. However, differences between treatments were not found statistically significant ( $p>0.05$ ) except for the differences between chick weights and liveability. Liveability of young breeder flocks were lower than old breeders in both first week and last week $(p<0.05)$. It was determined that liveability in rearing period was affected by breeder age whereas not affected by storage time and setter ventilation program (Table 5).

The chick weight differences of young and old flocks were high and significant $(p<0.05)$. But then they got closer in time and by the end of rearing period, average live weight and daily weight gain values were similar.

Table 5 - The effect of different incubation practices on liveability.

\begin{tabular}{|c|c|c|c|c|c|c|}
\hline & \multicolumn{6}{|c|}{ Liveability, \% } \\
\hline & 7th day & 14th day & 21st day & 28th day & 35th day & $\begin{array}{c}\text { 41st day } \\
\text { (Slaughter) }\end{array}$ \\
\hline \multicolumn{7}{|c|}{ Breeder Age (BA), week } \\
\hline 32, young $(Y)$ & $99.16 \pm 0.12^{a}$ & $98.59 \pm 0.16^{a}$ & $98.10 \pm 0.20^{a}$ & $97.88 \pm 0.24$ & $97.57 \pm 0.27$ & $96.94 \pm 0.35^{a}$ \\
\hline 55, old $(0)$ & $99.53 \pm 0.12^{b}$ & $99.06 \pm 0.16^{b}$ & $98.81 \pm 0.23^{b}$ & $98.62 \pm 0.29$ & $98.40 \pm 0.32$ & $98.96 \pm 0.31^{b}$ \\
\hline \multicolumn{7}{|c|}{ Storage Time (ST), day } \\
\hline 2 , short (S) & $99.35 \pm 0.13$ & $98.95 \pm 0.13$ & $98.56 \pm 0.22$ & $98.25 \pm 0.31$ & $98.06 \pm 0.33$ & $97.60 \pm 0.35$ \\
\hline 12, long $(\mathrm{L})$ & $99.34 \pm 0.13$ & $98.70 \pm 0.19$ & $98.35 \pm 0.24$ & $98.25 \pm 0.25$ & $97.91 \pm 0.30$ & $97.32 \pm 0.36$ \\
\hline \multicolumn{7}{|c|}{ Setter Ventilation Program (SVP) } \\
\hline Control (C) & $99.28 \pm 0.12$ & $98.69 \pm 0.18$ & $98.28 \pm 0.25$ & $98.00 \pm 0.30$ & $97.60 \pm 0.34$ & $97.00 \pm 0.38$ \\
\hline Test $(\mathrm{T})$ & $99.41 \pm 0.13$ & $98.94 \pm 0.19$ & $98.62 \pm 0.21$ & $98.50 \pm 0.25$ & $98.37 \pm 0.25$ & $97.90 \pm 0.28$ \\
\hline \multicolumn{7}{|c|}{ Breeder Age (BA) X Storage Time (ST) } \\
\hline YS & $99.38 \pm 0.18^{a b}$ & $98.88 \pm 0.18^{b}$ & $98.57 \pm 0.22^{b}$ & $98.19 \pm 0.41$ & $98.01 \pm 0.40$ & $97.51 \pm 0.49^{a b}$ \\
\hline YL & $88.94 \pm 0.11^{a}$ & $98.13 \pm 0.23^{a}$ & $97.63 \pm 0.23^{a}$ & $97.57 \pm 0.22$ & $97.13 \pm 0.31$ & $96.38 \pm 0.42^{a}$ \\
\hline OS & $99.31 \pm 0.18^{\mathrm{ab}}$ & $99.00 \pm 0.25^{b}$ & $98.56 \pm 0.41^{b}$ & $98.30 \pm 0.50$ & $98.12 \pm 0.55$ & $97.68 \pm 0.52^{\mathrm{ab}}$ \\
\hline $\mathrm{OL}$ & $99.75 \pm 0.09^{b}$ & $99.25 \pm 0.21^{b}$ & $99.16 \pm 0.22^{b}$ & $98.94 \pm 0.27$ & $98.69 \pm 0.34$ & $98.25 \pm 0.35^{b}$ \\
\hline \multicolumn{7}{|c|}{ Breeder Age (BA) X Setter Ventilation Program (SVP) } \\
\hline YC & $99.00 \pm 0.13$ & $98.25 \pm 0.13^{a}$ & $97.82 \pm 0.19$ & $97.44 \pm 0.29^{a}$ & $97.00 \pm 0.30^{\mathrm{a}}$ & $96.19 \pm 0.49^{a}$ \\
\hline YT & $99.32 \pm 0.19$ & $98.75 \pm 0.30^{\mathrm{ab}}$ & $98.38 \pm 0.33$ & $98.32 \pm 0.33^{\mathrm{ab}}$ & $98.13 \pm 0.36^{\mathrm{ab}}$ & $97.69 \pm 0.33^{\mathrm{ab}}$ \\
\hline OC & $99.56 \pm 0.15$ & $99.13 \pm 0.24^{b}$ & $98.75 \pm 0.40$ & $98.56 \pm 0.46^{b}$ & $98.19 \pm 0.55^{\mathrm{ab}}$ & $97.82 \pm 0.44^{\mathrm{ab}}$ \\
\hline OT & $99.50 \pm 0.19$ & $99.12 \pm 0.23^{b}$ & $98.87 \pm 0.27$ & $98.68 \pm 0.38^{b}$ & $98.62 \pm 0.35^{b}$ & $98.11 \pm 0.47^{b}$ \\
\hline \multicolumn{7}{|c|}{ Storage Time (ST) X Setter Ventilation Program (SVP) } \\
\hline SC & $99.25 \pm 0.16$ & $98.75 \pm 0.23$ & $98.25 \pm 0.34$ & $97.81 \pm 0.46$ & $97.63 \pm 0.51$ & $97.19 \pm 0.53$ \\
\hline ST & $99.44 \pm 0.19$ & $99.13 \pm 0.18$ & $98.87 \pm 0.27$ & $98.68 \pm 0.39$ & $98.50 \pm 0.38$ & $97.99 \pm 0.43$ \\
\hline LC & $99.31 \pm 0.19$ & $98.63 \pm 0.28$ & $98.32 \pm 0.38$ & $98.19 \pm 0.40$ & $97.57 \pm 0.48$ & $96.82 \pm 0.57$ \\
\hline LT & $99.38 \pm 0.18$ & $98.75 \pm 0.33$ & $98.38 \pm 0.32$ & $98.31 \pm 0.31$ & $98.25 \pm 0.35$ & $97.81 \pm 0.39$ \\
\hline \multicolumn{7}{|l|}{$p$ Value } \\
\hline BA & 0.031 & 0.045 & 0.026 & 0.056 & 0.055 & 0.036 \\
\hline ST & 0.995 & 0.297 & 0.515 & 0.991 & 0.735 & 0.585 \\
\hline SVT & 0.479 & 0.339 & 0.302 & 0.210 & 0.076 & 0.068 \\
\hline $\mathrm{BA} \times \mathrm{ST}$ & 0.007 & 0.008 & 0.010 & 0.095 & 0.082 & 0.047 \\
\hline BA X SVP & 0.095 & 0.041 & 0.088 & 0.097 & 0.049 & 0.019 \\
\hline ST X SVP & 0.894 & 0.565 & 0.534 & 0.490 & 0.358 & 0.309 \\
\hline
\end{tabular}

abc The difference between the averages indicated by different letters in the same column are statistically significant $(p<0,05)$. 
Also, it was found that interactions were significant $(p<0.05)$ between breeder age-storage time and breeder age-setter ventilation program for post-hatch performance (Table 6). Similarly, BW and DWG values were higher $(p<0.05)$ in chicks obtained from the eggs of young flocks placed to setter operated with normal ventilation program (control treatment) than the test program (accumulated $\mathrm{CO}_{2}$ ).

Table 6 - The effect of different incubation practices on post-hatch performance.

\begin{tabular}{|c|c|c|c|c|c|}
\hline & $C W, g$ & $\begin{array}{l}\text { BW, g } \\
41^{\text {st }} \text { day }\end{array}$ & DWG, g & $\begin{array}{c}F C R, \\
41^{\text {st }} \text { day }\end{array}$ & $\begin{array}{c}\text { EPEF, } \\
41^{\text {st }} \text { day }\end{array}$ \\
\hline \multicolumn{6}{|c|}{ Breeder age (BA), week } \\
\hline 32 (Young) & $39.32 \pm 0.19^{b}$ & $2,302 \pm 5$ & $55.20 \pm 0.12$ & $1.778 \pm 0.006$ & $306.27 \pm 2.14$ \\
\hline 55 (Old) & $47.42 \pm 0.11^{\mathrm{a}}$ & $2,304 \pm 10$ & $55.05 \pm 0.25$ & $1.772 \pm 0.010$ & $310.81 \pm 2.35$ \\
\hline \multicolumn{6}{|c|}{ Storage Time (ST), day } \\
\hline 2 & $43.63 \pm 0.99$ & $2,310 \pm 8$ & $55.29 \pm 0.20$ & $1.774 \pm 0.005$ & $309.97 \pm 1.62$ \\
\hline 12 & $43.12 \pm 1.12$ & $2,297 \pm 8$ & $54.97 \pm 0.19$ & $1.776 \pm 0.011$ & $307.10 \pm 2.81$ \\
\hline \multicolumn{6}{|c|}{ Setter Ventilation Program (SVP) } \\
\hline Control (C) & $43.33 \pm 1.08$ & $2,304 \pm 6$ & $55.13 \pm 0.15$ & $1.775 \pm 0.009$ & $307.28 \pm 2.23$ \\
\hline Test (T) & $43.41 \pm 1.03$ & $2,303 \pm 10$ & $55.11 \pm 0.24$ & $1.776 \pm 0.007$ & $309.80 \pm 2.37$ \\
\hline \multicolumn{6}{|c|}{ Breeder Age (BA) X Storage Time (ST) } \\
\hline Young 2days (YS) & $39.84 \pm 0.25^{b}$ & $2,311 \pm 6^{a}$ & $55.40 \pm 0.15$ & $1.767 \pm 0.006$ & $311.10 \pm 1.95$ \\
\hline Young 12days (YL) & $38.80 \pm 0.08^{b}$ & $2,294 \pm 7^{b}$ & $54.99 \pm 0.18$ & $1.789 \pm 0.010$ & $301.45 \pm 3.02$ \\
\hline Old 2 days (OS) & $47.42 \pm 0.15^{a}$ & $2,309 \pm 15^{a b}$ & $55.17 \pm 0.38$ & $1.781 \pm 0.008$ & $308.85 \pm 2.66$ \\
\hline Old 12days (OL) & $47.43 \pm 0.17^{a}$ & $2,299 \pm 15^{a b}$ & $54.93 \pm 0.36$ & $1.763 \pm 0.018$ & $312.76 \pm 3.95$ \\
\hline \multicolumn{6}{|c|}{ Breeder Age (BA) X Setter Ventilation Program (SVP) } \\
\hline Young Control (YC) & $39.17 \pm 0.14^{b}$ & $2,313 \pm 6^{a}$ & $55.47 \pm 0.15^{a}$ & $1.780 \pm 0.007$ & $305.03 \pm 2.91$ \\
\hline Young Test (YT) & $39.47 \pm 0.35^{b}$ & $2,292 \pm 7^{b}$ & $54.93 \pm 0.16^{b}$ & $1.776 \pm 0.010$ & $307.51 \pm 3.27$ \\
\hline Old Control (OC) & $47.50 \pm 0.12^{a}$ & $2,294 \pm 8^{\mathrm{ab}}$ & $54.80 \pm 0.20^{\mathrm{ab}}$ & $1.770 \pm 0.018$ & $309.53 \pm 3.38$ \\
\hline Old Test (OT) & $47.35 \pm 0.19^{a}$ & $2,315 \pm 19^{\mathrm{ab}}$ & $55.30 \pm 0.47^{\mathrm{ab}}$ & $1.775 \pm 0.010$ & $312.08 \pm 3.44$ \\
\hline \multicolumn{6}{|c|}{ Storage Time (ST) X Setter Ventilation Program (SVP) } \\
\hline 2days Control (SC) & $43.59 \pm 1.56$ & $2,313 \pm 9$ & $55.34 \pm 0.23$ & $1.775 \pm 0.007$ & $308.88 \pm 1.97$ \\
\hline 2 days Test (ST) & $43.67 \pm 1.33$ & $2,308 \pm 14$ & $55.23 \pm 0.34$ & $1.774 \pm 0.008$ & $311.07 \pm 2.65$ \\
\hline 12 days Control (LC) & $43.08 \pm 1.60$ & $2,295 \pm 6$ & $54.93 \pm 0.17$ & $1.774 \pm 0.018$ & $305.69 \pm 4.09$ \\
\hline 12days Test (LT) & $43.16 \pm 1.67$ & $2,298 \pm 16$ & $55.00 \pm 0.36$ & $1.778 \pm 0.012$ & $308.52 \pm 4.07$ \\
\hline \multicolumn{6}{|l|}{$p$ Value } \\
\hline BA & 0.000 & 0,867 & 0.601 & 0.622 & 0.164 \\
\hline ST & 0.736 & 0,237 & 0.255 & 0.865 & 0.386 \\
\hline SVP & 0.959 & 0,945 & 0.940 & 0.925 & 0.445 \\
\hline $\mathrm{BA} \times \mathrm{ST}$ & 0.000 & 0,679 & 0.653 & 0.353 & 0.058 \\
\hline$B A X S V P$ & 0.000 & 0,341 & 0.297 & 0.942 & 0.481 \\
\hline ST X SVP & 0.990 & 0,689 & 0.713 & 0.994 & 0.724 \\
\hline
\end{tabular}

CW: Chick Weight, BW: Body Weight, DWG: Daily Weight Gain, FCR: Feed Conversion Ratio, EPEF: European Production Efficiency Index ${ }^{a b}$ The difference between the averages indicated by different letters in the same column are statistically significant $(p<0,05)$.

\section{DISCUSSION}

This research was designed to obtain higher incubation and growing performance and to reduce the deviation in performance by determining the effects of breeder age, storage time and setter ventilation program. Embryonic deaths and fertile hatchability of fertile eggs (HF) for incubation performance, liveability, and chick weight (CW), body weight (BW), daily live weight gain (DWG), feed conversion ratio (FCR) and European Productivity Index (EPEF) values for growing performance were evaluated.
ED of young flocks and accordingly HF was higher than older flock eggs. Similarly, lower early stage embryonic deaths and late stage embryonic deaths plus pipped and unhatched embryo rates and accordingly higher hatchability of fertile eggs in short time stored eggs have been obtained from experimental data. In contrast, incubation performances of eggs incubated in setters operated to different ventilation program were similar. Also, depending on the important interactions between treatment groups. It has been found that ED and HF were improved, when hatching eggs of old flocks incubated in setter operated to by accumulated $\mathrm{CO}_{2}$. 
The results of this study supported the findings of Mc Daniel (1995) showing that early and late stage embryonic mortality is 3-5\% and this seems to be consistent with the general statement in this regard. The results of setter ventilation program trial in this study was supported by researches (Ross Tech, 2003) showing that setter accumulated $\mathrm{CO}_{2}$ ventilation program has positive effects and negative effects have not been identified yet.

In terms of post-hatch performance, differences between treatments were not found significantly different ( $p>0.05)$ except for the difference between chick weights and liveability $(p<0.05)$. Liveability of young breeder flocks were lower in both the first week and slaughter. But, differences between CW values during the rearing period and BW and DWG values of treatment groups were found to be similar at the end of growing period. In addition, higher chick weight, BW and DWG values have been achieved in eggs of YC than YT.

These results were supported by data from Christensen et al. (1996) that liveability decreased with flock age and storage. Also, liveability data seems to be consistent with the data of (Bowling \& Howarth, 1981; Elibol, 1997) who observed live weight differences in the first week and also closed up later in the rearing period. Similarly, data showing impairment in live weight are supported in terms of flock age (Christensen et al., 1996), but data suggesting deterioration in feed conversion ratios are supported (Hill, 2002). In contrast, unlike the incubation performance, in terms of growing performance, similar results were obtained from the study showing that raising $\mathrm{CO}_{2}$ levels to a certain level or operating the $\mathrm{CO}_{2}$ control system has negative effect on post-hatch performance (Taylor, 2000).

As a result, higher incubation and post-hatch performance were obtained from the eggs of young flocks stored for a short period. In addition, it was found that incubation and growing period performances of long time stored old flock eggs can be improved by incubating with accumulated $\mathrm{CO}_{2}$ ventilation program. Thus, a more healthy and with higher quality chick production can be achieved and consequently production costs can be reduced and the competiveness of poultry companies can be improved.

\section{ACKNOWLEDGMENTS}

I thank my college professors for the encouragement to develop this study and Beypi Inc. (beypiliç)'s General Manager Dr. SaitKoca for support in every stage.

\section{REFERENCES}

AOAC. Official method of analysis. $15^{\text {th }}$ ed. Washington: Association of Official Analytic Chemist; 1990.

Barrot HG. Effect of temperature, humidity and other factors on hatch of hen's eggs and on energy metabolism of chick embryos. USDA Technical Bulletin 1937;553:1-45

Becker WA, Spencer JV, Swartwood JL. Carbon dioxide during storage of chicken and turkey hatching eggs. Poultry Science 1968;47:251-258.

Boerjan M. Uniform incubation. Netherland: Pass Reform Hatchery Technologies; 2011

Bowling JA, Howarth B. The effect of broiler breeder eggs exposing to high temperature before storage on hatchability and subsequent performance of chicks. Poultry Science 1981;60:2333-2336.

Bruzal, JJ, Peak SD, Peebles ED. Effects of relative humidity during incubation on hatchability and body weight of broiler chicks from young breeder flocks. Poultry Science 2000;79:827-830.

Butcher G. Techniques for embryo-diagnosis and development of the chicken embryo. ASA Annual Conference; 2004; Duhram. Inglaterra.

Christensen VL, Donalson VL, Mc Murty JP. Physiological differences in late embryos at different ages. Poultry Science 1996;75(2):172-178.

Dawes $C$, Simkiss $K$. The effects of respiratory acidosis in the chick embryo. Journal of Experimental Biology 1971;55:77-84.

De Smit L, Bruggeman V, Debonne M, Tona JK, Kamers B, Everaert N, et al. The effect of nonventilation during early incubation on the embryonic development of chicks of two commercial broiler strains differing in ascites susceptibility. Poultry Science 2008;87:551-560.

De Smit L, Bruggeman V, Tona JK, Debonne M, Onagbesan O, Arckens $L$, et al. Embryonic developmental plasticity of the chick:increased $\mathrm{CO}_{2}$ during early stages of incubation changes the developmental trajectories during prenatal and postnatal growth. Comparative Biochemistry and Physiology Part A 2006;145:166-175.

Duncan DB. Multiple range test for multiple moment. Biometrics 1955;11:142.

Elibol O, Brake J. Effect of frequency of turning from three and eleven days of incubation on hatchability of broiler eggs. Poultry Science 2003:82:357-359.

Elibol O, Turkoglu M. Embryo development and hatchery. In:Turkoglu M, Sarica M, editors. Poultry scince. $4^{\text {th }}$ ed. Ankara: BeyOfset; 2014 p.200-206.

Elibol O. Clearing incubation and incubation factors affecting results of failures and research on determination [thesis). Ankara (TUR): University Institute of Science and Technology; 1997.

Everaert N, Kamers B, Witters A, De Smit L, Debonne M. Decuypere E et al. Effect of four percent carbon dioxide during the second half of incubation on embryonic development, hatching parameters and posthatch growth. Poultry Science 2007;86:1372-1379.

Everaert N, Willemsen $\mathrm{H}$, De Smit L, Witters A, De Baerdemaeker J, Decuypere $\mathrm{E}$, et al. Comparison of a modern broiler and layer strain during embryonic development and the hatching process. British Poultry Science 2008;49:574-582

Fares WA, Shahein EHA, Rizk RE, El-Hanoun AM. Carbon dioxide as affected by ventilation process during early stage of incubation and its relation with embryonic development, hormone levels, hatching parameters and post-hatch chicks' growth. Egyptian Poultry Science 2011;32(1):23-41 
Gildersleeve RP, Boeschen DP. The effect of incubator carbon dioxide on turkey hatchability. Poultry Science 1983;62:779-784.

Hill D. Performance loses: incubation and brooding. East Riding of Yorkshi: International Hatchery Practice; 2002. p.1-8.

Hodgetts B. Determination of incubation failures and remedies. Proceedings of YUTAV International Poultry Congress; 1993; Istanbul. Turkey. p.303-309.

Hogg A. Single stage incubation trials. Poultry and Avian Biology Review 1997;8:168.

Mather CM, Laughlin KF. Storage of hatching eggs: the effect on total incubation period. British Poultry Science 1976;17:471-479.

Mather CM, Laughlin KF. Storage of hatching eggs:the interaction between parental age and early embryonic development. British Poultry Science 1979;20:595-604.

Mc Daniel GR. Hatching failure and solutions. Proceedings of the YUTAV International Poultry Exhibition and Conference; 1995; Istanbul. Turkey. p.58-61.

NRC. Nutrient requirements of poultry. $9^{\text {th }}$ ed. Washington: National Research Council; 1994.

Onagbesan O, Bruggeman V, De Smit L, Debonne M, Witters A, Tona K, et al. Gas exchange during storage and incubation of avian eggs:Effects on embryogenesis, hatchability, chick quality and post-hatch growth. World Poultry Science Journal 2007;63:557-573.

Quintana JA, Silva M, Ruiz R, Cazares R, Merino R. Influence of hatching egg and broiler chicken storage on hatchability performance. Proceedings of the 21th World Poultry Congress; 2000 Aug 20-24; Montreal, Quebec. Canada: Poultry Science Association; 2000.

Reis LH, Gamma T, Soares MC. Effects of short storage conditions and broiler breeder age on hatchability, weights chick hatching time. Poultry Science 1997;76:1459-1466.

Romanoff AL. Critical periods and causes of death in avian embryonic development. The Auk 1949;66:264-270.

Romanoff AL, Romanoff AJ. Biochemistry and biophysics of the developing hen's egg: ii. Influence of composition of air [bulletin 160]. Ithaca: Cornell University, Agricultural Experimental Station; 1933. p.1-36.
Ross broiler: nutrition specification [Ross 308]. Midlothian: Aviagen, 2007.

Ross Tech. Investigating hatchery practice [document 98/35]. Midlothian: Aviagem; 2003.

Salamon A, Kent JP. Changes during incubation within double-yolked duck (Anasplatyrhynchosdomesticus) eggs: yolk position, mortality, hatchability and the importance of an optimal egg size. International Journal of Poultry Science 2014;13:695-702.

SPSS. IBM SPSS statistics for windows. Version 22.0. Armonyk: IBM Corp; 2013.

Taylor G. High-yield breeds require special incubation. World's Poultry Science Journal 1999;3(15):27-29.

Tona K, Everaert N, Willemsen $\mathrm{H}$, Gbeassor M, Decuypere E, Buyse J. Effects of interaction of incubator $\mathrm{CO}_{2}$ levels and mixing hatching eggs of different embryo growth trajectory on embryo physiological and hatching parameters. British Poultry Science 2013;54(4):545-551.

Tona K, Onagbesan O, Bruggeman V, De Smit L, Figueiredo D, Decuypere E. Non-ventilation during early incubation in combination with dexamethasone administration during late incubation:1. Effects on physiological hormone levels, incubation duration and hatching events. Domestic Animal Endocrinology 2006;33(1):32-46.

Tona K, Onagbesan O, Bruggeman V, De Smit L, Figueiredo D, Decuypere E. Non-ventilation during early incubation in combination with dexamethasone administration during late incubation. 1. Effects on physiological hormone levels, incubation duration and hatching events. Domestic Animal Endocrinology 2007;33:32-46.

Tona K, Onagbesan O, Ketelaere B, Decuypere E, Bruggeman V. Effects of age of broiler breeders and egg storage on egg quality, hatchability, chick quality, chic weight and chick post hatch growth to forty-two days. Journal of Applied Poultry Research 2004;13:10-18.

Turkoglu M, Sarica M, Altan A, Arda M, Bayraktar H, Kutlu H, et al. Poultry science: growing, nutrition, diseases. Ankara: BeyOfset Press; 2014.

Willemsen $\mathrm{H}$, Tona K, Bruggeman V, Onagbesan O, Decuypere E. Effects of high $\mathrm{CO}_{2}$ level during early incubation and late incubation in ovo dexamethasone injection on perinatal embryonic parameters and posthatch growth of broilers. British Poultry Science 2008;49(2):222-231. 
